\title{
A COMPARATIVE STUDY BETWEEN THE RUNNING OF THE FIRST AND SECOND CURVES IN SOME BIOMECHANICAL INDICATORS TO PASS BARRIERS AT THE EFFECTIVENESS OF $400 \mathrm{M} / \mathrm{BARRIERS}$
}

\author{
*Ali Naim Ajil, **Prof.Dr. Sareeh Abdul Karim Al-Fadhli \\ *University of Wasit / College of Physical Education and Sports Sciences \\ **University of Baghdad / College of Physical Education and Sports Sciences
}

DOI: $10.37648 / \mathrm{ijrssh.v10i02.030}$

Received: 30 ${ }^{\text {th }}$ March, 2020; Accepted: 20 th April, 2020; Published: $13^{\text {th }}$ May,2020

\begin{abstract}
The most important thing that affects the race time is the availability of special physical abilities as well as the external forces affecting the running track of the runner. One of these forces is the centrifugal force because the effectiveness of 400 $m$ / barriers contain two curves, so this strength is a great indication of the race time, where it must be The length and frequency of the steps in the two curves are ideal to a large extent in the face of this force compared to the length and frequency of the steps in the rectum as well as maintaining them between the barriers and the fit of these steps with the barrier step, which must be consistent with the rest of the steps as well as the nature of the upgrading of the left leg to the right foot, so that it secures No decrease in speed or uniform The length of the steps is more than normal, especially when passing through the barriers in the curves, where the focus must be on training the runners physically and skillfully greatly to control the length of the step and its frequency, hence the importance of research through finding comparison aspects between the ratios of some biomechanical indicators between the two curves to enable in the future to develop An appropriate business plan, whether corrective at the skill level or training at the physical level so that the physical and mechanical variables in the curve are in their ideal shape, the purpose of the researchers from the study:

- A comparative study between the running of the first and second curves in some biomechanical indicators to pass barriers in the effectiveness of $400 \mathrm{~m} /$ barriers.

- As for the research tests, they were as follows: / Achievement test for the effectiveness of 400 meters hurdles.

- Measuring the average velocity of the first and second curves during the performance of the first test, the second first test, finding the centrifugal force rate at the top of the two curves.
\end{abstract}

Keywords: Centrifugal law, biomechanical indicators, curves first and second, $400 \mathrm{~m}$ hurdles. 


\section{INTRODUCTION}

The achievement of achievement and obtaining a high level in athletics activities does not come through the use of the best scientific methods in sports training only, but rather as a result of proper use of modern measurements and tests and scientific planning accompanied by the results of tests for movement laws and their practical application in sports training as well as focusing on training parts And effectively divide the activity.

The effectiveness of $400 \mathrm{~m} /$ hurdles is one of the activities that depend on high-precision technique on the barriers and maintaining the special physical capabilities for the length of the race period, which requires hostility physical and skill integration in order to maintain the same rhythm in all parts of the race without a clear decrease in the length and frequency of the step.

The most important thing that affects the race time is the extent to which the level of biomechanical indicators between the two curves coincide. , Where the length and frequency of the steps in the curves should be ideal to a large extent in the face of this force compared to the length and frequency of the steps in the rectum as well as maintaining them between the barriers and the fit of these steps with the barrier step, which must be consistent with the rest of the steps as well as the nature of the upgrade of a man to $\mathrm{R}$ man right so as to ensure not decreasing speed or increasing the length of more steps than normal, especially when passing the barriers in Almnhanin, where you must focus on the training of runners physically and Mharria significantly to control the step length and frequency and thus loss of time through curves as a result of an act of centrifugal force.

From here, the importance of research lies by finding comparative aspects between the ratios of some biomechanical indicators between the two curves to enable in the future to develop an appropriate work plan, whether it is corrective at the skill level or training at the physical level so that the physical and mechanical variables in the curve are ideal.

In a study by (Ahmed Nagy and Intisar Hameed 2012), emphasis is placed on training players according to biomechanical indicators to develop momentum moments at each stage and reduce speed loss between the two stages in order to improve achievement in general.

In a study of (Han, 2018) whose results indicated the necessity of emphasizing the training of curves as it constitutes a natural resistance to the runner as a result of the effect of the centrifugal force which in turn will pull the gallop outward while the runners will try to use the force of the pull and as a result this will definitely affect the speed of the gallop.

So the purpose of the study was:

-A comparative study between the running of the first and second curves in some biomechanical indicators to pass barriers in the effectiveness of $400 \mathrm{~m} /$ barriers.

\section{MATERIALS AND METHODS:}

The researchers relied on the use of the descriptive method in the comparison method to solve the research problem 'as the research sample was chosen intentionally for the availability of conditions and ease of implementation of field procedures as the research sample consisted of (6) runners for the effectiveness of running $400 \mathrm{~m}$ / barriers within the center of sports talent in Baghdad and the researchers used devices and auxiliary tools And the means of collecting the following information:

400m barriers / barriers number 10 -

- Computer

- Funnel Number 30

- Arab and foreign sources

- International information web

The exploratory experiment was conducted in the stadium for athletics in the Ministry of Youth and Sports and its purpose in order to identify the difficulties that are encountered when applying the research experience. In addition, to know the adequacy of the auxiliary work team and the duties assigned to them when conducting the tests, the researchers conducted the tribal tests in the middle of October The first of 2019 and on the track of athletics in the Ministry of Youth and Sports, the following test was applied:

Achievement test to run the effectiveness of 400 meters hurdles

Measuring the average velocity of the first and second curves during the performance of the first test, the first test, the second, finding the rate of centrifugal force at the top of the two curves.

After completing the application of the test, the researchers statistically processed the data and intentionally analyzed and interpreted the results according to the levels of the physical sample, where they calculated the total distance time through the lowest possible time until the finish line and the average velocity 
of each curve was extracted as well the calculation of the central torque force at the highest peak of the curve and that By deducting a distance of $5 \mathrm{~m}$ and calculating its RESULT AND DISCUSSION: speed, knowing that the radius of the curve is known and the following law has been applied:

Attractive or repulsive force $=\mathrm{K} \times 2 / \mathrm{Naq}$

\begin{tabular}{|l|l|l|l|l|l|}
\hline $\begin{array}{l}\text { Level of } \\
\text { significance }\end{array}$ & $\begin{array}{l}\text { The level of } \\
\text { confidence }\end{array}$ & Calculated t & \multicolumn{2}{|l|}{ Arithmetic mean } & Variables \\
\hline moral & 0.003 & 3.863 & 16.70 & Curve 1 & $\begin{array}{l}\text { Average } \\
\text { speed }\end{array}$ \\
\cline { 3 - 5 } & & & 19.78 & Curve 2 & \\
\hline moral & 0.004 & 2.658 & 15.69 & Curve 1 & $\begin{array}{l}\text { Average } \\
\text { speed }\end{array}$ \\
\cline { 4 - 5 } & & & 18.72 & Curve 2 & \\
\hline
\end{tabular}

Through table (1) it is clear from the arithmetic mean that there is a weakness in the arithmetic circles of the second curve from the first curve. This is clear evidence of weak physical abilities and a weak level of skill performance for the runner, whether in the mechanism of running the distance or in the ability to traverse quickly and effectively and all this as a result of weakness in The ability to bear speed as well as endure which requires the trainer to activate the role of speed bearing in training units, and this has a positive impact in improving their training efficiency, which gives them the ability to run this distance at a level of retaining the speed rates within the distance limits, in addition to that the sample is printed on running along the step and is shown Stable according to the runner's sense of the target time as a result of the consistency of the training according to the speed curve of the second curve from the first, which leads to the printing of the runner to make the length of the step and its frequency according to the average of the speed of the second curve compared to the first and also must simulate the biomechanical role of the training process by mixing the training process with mechanical laws that support the process Training with laws that do not accept doubts about their positive outcomes, since the law of centrifugation must be applied in terms of severity, training size, and rest, according to the maximum stresses obtained through the rate of centrifugal force, which are compatible with the bearing requirements The speed of this activity is with maximum and subacute repetitions, which leads to the normalization of the muscles working on performance in a high way and this is what both (Al-Ela and Allawi) referred to. In the body systems and the rise in the level, so it is one of the most important factors for success in the training program and thus in improving performance.

"The ability of hostility develops when training to the pace of the steps during the distance training exercises for the runners at a 400 meter hurdles runner, as the runner tries to maintain the best rate of speed during the later stages of the race and thus they contributed to the reduction of time."

\section{REFERENCES:}

- Frank Abdul Karim (2018-2019) speed limiters and their practical applications in physical effort (part 2), a documented lecture on PhD students Baghdad University / College of Physical Education and Sports Science.

- Muhammad Hassan Allawi, Abu Al-Ella Ahmed: Physiology of Athletic Training, Cairo, Dar Al-Fikr Al-Arabi, 1984.

- Sabah Mahdi Salih (2013) Biomechanical variables for the fields of the enemy curve $200 \mathrm{~m}$ and the percentage of its contribution to speed and achievement, Master Thesis, University of Qadisiyah, College of Physical Education.

- Salah Mohsen Naga, (1998) Athletics, Basis, Education, Organization, Cairo, Asr Language Center for Computers and Printing. 